\title{
The Comparison of Template Matching and SURF for Logo Classification on Product
}

\author{
Thummarat Boonrod $^{\mathrm{a}^{*}}$, Chatklaw Jareanpon ${ }^{\mathrm{a}}$, Phatthanaphong Chomphuwiset ${ }^{\mathrm{a}}$ \\ ${ }^{a}$ Department of Computer Science, Mahasarakham University, \\ Kantarawichai District, Maha Sarakham, Thailand \\ *Corresponding Author: thummaratboon@gmail.com
}

\begin{abstract}
This paper proposes the fast logo classification on product. The search space for logo classification is reduced by production using Histogram of Oriented Gradients (HOG). The template matching and Speeded-Up Robust Features (SURF) algorithm are used to detect the logo, and in term of the computational time. The experimental results found that the HOG can be detected the area and product at $88.00 \%$ accuracy rate. The logo detection found that the advantage of template matching is simple, but the advantage of SURF algorithm is speed.
\end{abstract}

Keywords: Histogram of Oriented Gradients (HOG), Template matching, Speeded-Up Robust Features (SURF).

\section{Introduction}

The logo is graphic or symbol that represents the company, organization, product, activity, or technology. The logo design is often represented by letter, word, image, or combinations. The objective of the logo design is easy for recognition, unique, and attracting with the customer. The logo is used to easily recognize instead of text. ${ }^{(1,2)}$ Nowadays, the logo is used in various fields such as logo classification in vehicle for identifying. ${ }^{(3)}$ Logo in product detection system helps for the product description. Logo detection and classification has a challenging issue in topics of background cluttered, brightness, size, and computational time. Many researches proposed the various solutions for logo detection and classification using SIFT algorithm. However, The SIFT algorithm uses high memory and time-consuming. Kalaiyarasi. et al. proposed the logo detection from video capturing using the PCA-SIFT (principal component analysis SIFT) method by improving
Scale Invariant Feature Transform (SIFT) to increase the speed. The normalized gradient is used by PCA to describe the interested point and the speed. ${ }^{(4)}$ Juan. and Gwun proposed the comparison of the computational time between SIFT, PCA-SIFT and SURF. The research found that the computational time of SUFT is the best. ${ }^{(5)}$

This paper proposes the fast logo classification on product consisted of 2 processes, product detection and logo classification. The product detection used to reduce the space by only selected in product area. That is possible to detect the outside product logo. Template matching and SURF algorithm are used for logo classification. The advantage of template matching is simple, but the advantage of SURF algorithm is speed. The next sections of this paper are organized as follow: section 2 is the related work, section 3 explains the about methodology, section 4 shown the experimental results, section 5 discusses the conclusions, and section 6 is the future enhancement.

\section{Related Work}

\subsection{Logo Design for Product ${ }^{(6)}$}

The logo of products is the symbols or markers created by human. These symbols are recognizable and simple. The logo are divided into 4 types, (a) Letter mark, (b) Word mark, (c) Pictorial symbol, and (d) Combinational mark.

(a) Letter mark

The letter mark is the logo design used from letters or acronyms that are modified for beauty as shown in figure 1 .

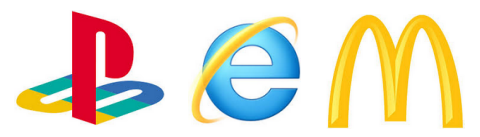

Fig. 1. letter mark logo design. 
(b) Word mark

The word mark is the logo design used from words or texts as shown in figure 2 .

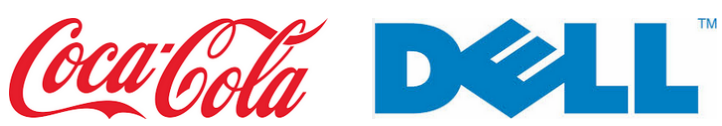

Fig. 2. word mark logo design.

(c) Pictorial symbol

The pictorial symbol is the logo design used from images or symbols that are abstract as shown in figure 3 .
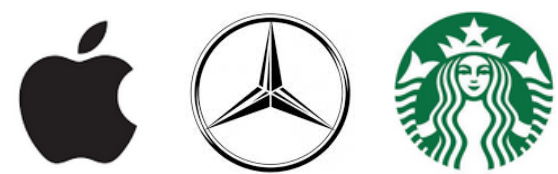

Fig. 3. pictorial symbol logo design.

(d) Combinational mark

The combination mark is the combination of logo design used from the letter, word, image, or symbols as shown in figure 4 .
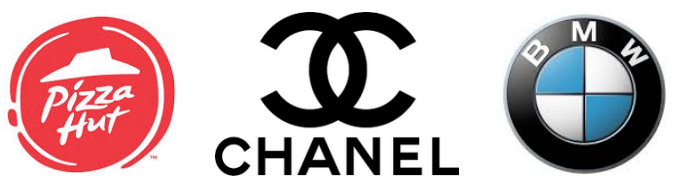

Fig. 4. Combinational mark logo design.

Moreover, the logo position on product has very important to recognize. Teng. proposed the experiment of human eye motion. This results found the similarity of the behavior of human vision by the movement pattern eyes. The order of the movement of the eyes are shown in figure 5 , starting from point number $1 .^{(7)}$

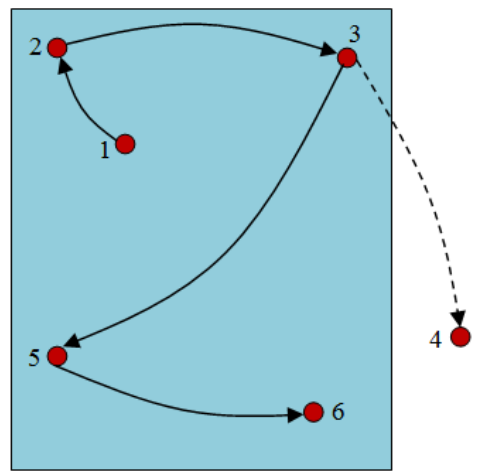

Fig. 5. The order of the movement of the eyes.

\subsection{Histogram of Oriented Gradients (HOG) ${ }^{(8)}$}

Histogram of Oriented Gradients (HOG) is the feature extraction method that is stable for a cluttered background and brightness. HOG is able to use to extract the feature of object in the region based on distribution of oriented gradient. Feature extraction is divided the region of the image into the small parts called cells and group of cells called block. The process of HOG is consisted of 4 processes, (a) Gradient computation, (b) Orientation binning, (c) Descriptor blocks, and (d) Block normalization.

(a) Gradient computation

The Gradient computation can be calculated from the vector $\mathrm{x}$-axis and $\mathrm{y}$-axis, and convoluted with the kernel filter as shown in figure 6 .

\begin{tabular}{|l|l|l|}
\hline-1 & 0 & 1 \\
\hline
\end{tabular}

Gx

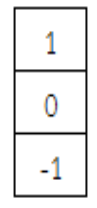

Gy
Fig. 6. Kernel filter.

The magnitude of gradient and orientation gradients are calculated from the equation (1) and equation (2).

$$
\begin{aligned}
& |G|=\sqrt{G x^{2}+G y^{2}} \\
& \theta=\arctan \frac{G x}{G y}
\end{aligned}
$$

when $\quad|G| \quad$ is gradients.

$G x^{2} \quad$ is second-order derivative in x-axis

$G y^{2} \quad$ is second-order derivative in y-axis

(b) Orientation binning

Each cell creates the histogram, and the value of gradients is stored to the bin. The orientation defined from $0-180$ or $0-360$ degree is categorized to the 9 bins of histogram.

(c) Descriptor blocks

The descriptor blocks are the feature description from all blocks in the form of the vectors.

(d) Block normalization

This process is normalization by the equation (3).

$$
\text { L1-norm: } f=\frac{v}{\sqrt{\|v\|_{k}+e}}
$$

when

$v$ is non-nomalized.

$k \quad$ is values 1 .

$e \quad$ is small constant value. 


\subsection{Template matching ${ }^{(9)}$}

Template matching is the identification the template with the image by finding the maximum value of correlation as shown in figure 7.

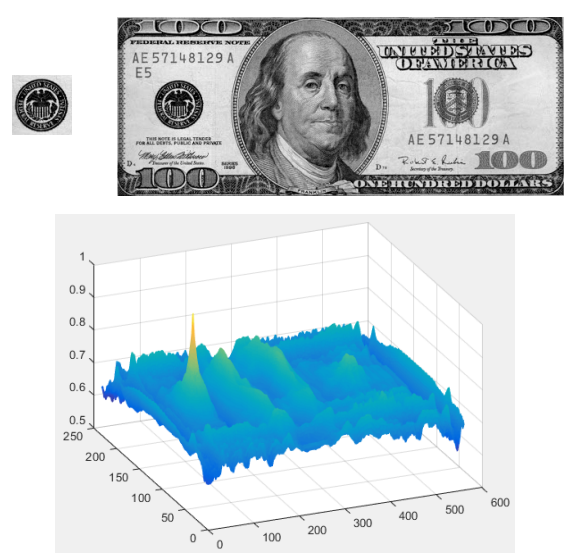

Fig. 7. The maximum of correlation.

The similar measurement of template matching is decided by Normalized Cross-Correlation (NCC) and calculated by the equation (4).

$$
C(f, g)=\frac{n \sum f g-\sum f \sum g}{\sqrt{\left(n \sum f^{2}-\left(\sum f\right)^{2}\right)\left(n \sum g^{2}-\left(\sum g\right)^{2}\right)}}
$$

when $n$ is the number of pixels in the template.

$f$ is image template.

$g \quad$ is image part under the templates.

\subsection{Speeded-Up Robust Features (SURF) ${ }^{(10)(11)}$}

Speeded-Up Robust Features (SURF) is the feature extraction that speeds up the process and robust to change of the image (size, rotation, blur and brightness). Feature extraction is consisted of 2 processes. (a) Interested point detection, and (b) Interest point description.

(a) Interested point detection

Interested point detection is used for the detection of interested region. This process is consisted of 4 processes.

(i) Integral Image is the calculation of the sum of the pixels which is help to reduce the computational time calculated by the equation (5).

$$
I_{\Sigma}(X)=\sum_{i=0}^{i \leq x} \sum_{j=0}^{j \leq y} I(i, j)
$$

When $I_{\Sigma}(X) \quad$ is integral image position $X=(x, y)$

$i \quad$ is row.

$j \quad$ is column (ii) The Hessian matrix is the area of interested point in the image calculated by the equation (6).

$$
H(X, \sigma)=\left[\begin{array}{ll}
L_{x x}(X, \sigma) & L_{x y}(X, \sigma) \\
L_{x y}(X, \sigma) & L_{y y}(X, \sigma)
\end{array}\right]
$$

when $L_{x x}(X, \sigma), L_{x y}(X, \sigma), L_{y y}(X, \sigma)$ are the second-order derivative of Gaussian image I position X.

(iii) Scale space is the pyramid created by the convolution image with the Gaussian kernel. The box filter is various sizes such as $9 \times 9,15 \times 15,21 \times 21,27 \times 27$, and it is used for each different size without changing the physical size of the image as shown in figure 8.

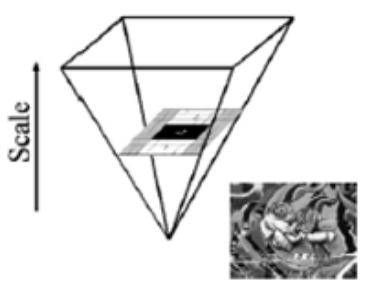

Fig. 8. Scale space is used box filter. ${ }^{(10)}$

(iv) The interested point is the points that are robust to change of image using Non-maximal suppression. The interested point is the comparison of the 26 pixels adjacent at the same, above and below layers. If the central point is greater than the all neighbors points, it will select as an interesting point. Conversely, it will reject as shown in figure 9 .

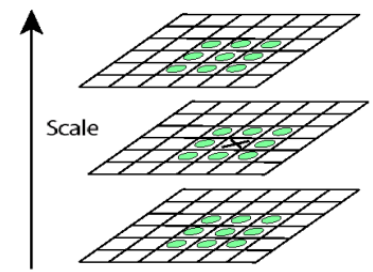

Fig. 9. The comparison of the adjacent pixels. ${ }^{(10)}$

(b) Interested point description

Interested point description is assigned by the orientation. This process is consisted of 2 processes.

(i) Orientation assignment is using Haar wavelets filter for the robust rotation to identify the direction of the interested point. Haar wavelets filter used the gradient in the $\mathrm{x}$-axis and the axis $\mathrm{y}$ as shown in figure 10 .

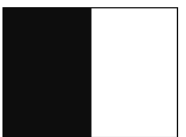

Gx

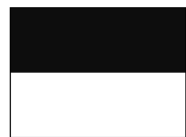

Fig. 10. Haar wavelets filter. ${ }^{(10)}$ 
(ii) Descriptor based on sum of Haar wavelet is the created square windows covering the key points and assigns the assignment orientation as shown in figure 11.

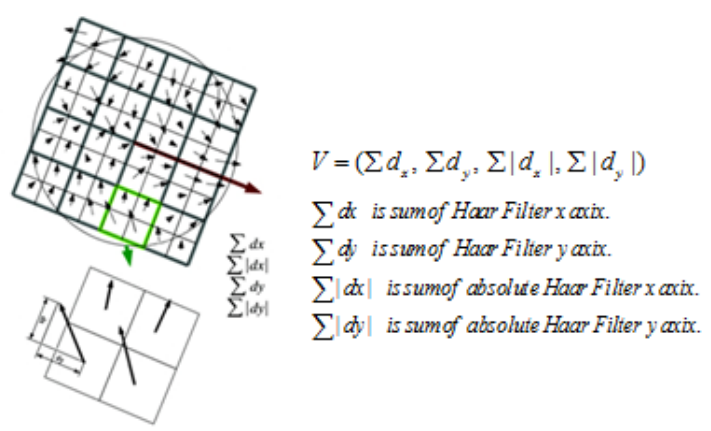

Fig. 11. Orientation of key point. ${ }^{(10)}$

\subsection{Support Vector Machine (SVM) $)^{(12)(13)}$}

Support Vector Machine (SVM) is the supervised learning used for classification. The plane called hyperplane is divided the data into 2 groups as shown in figure 12(a). The distance between 2 groups called margin away as much as possible. The contact data at the margin called support vector are shown in figure 12(b).

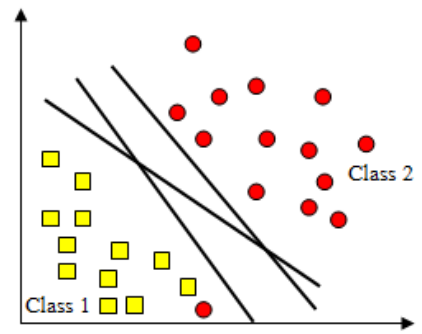

(a) The plane to try divides 2 groups. ${ }^{(12)}$

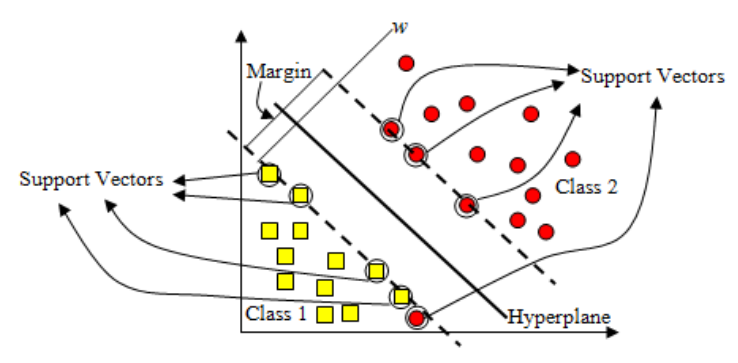

(b) The plane to divides the data into 2 groups. ${ }^{\text {(13) }}$

Fig. 12. Support Vector Machine

The learning datasets are $D=\left\{\left(\vec{x}_{i}, y_{i}\right)\right\}$ by that $\vec{x}_{i}\left(w_{i 1}, w_{i 2}, w_{i 3}, \ldots, w_{i m}\right)$ is input vector used to define the class $y_{i}$ when $y_{i}$ is the real numbers, $(-1$ to +1$)$. The

hyperplane is a line that the value of $y_{i}$ is equal to 0 by the equation (7).

$$
y=\left\{\begin{array}{l}
+1, \vec{w} \cdot \vec{x}+b>0 \\
-1, \vec{w} \cdot \vec{x}+b<0
\end{array}\right.
$$

when $\quad \vec{w} \quad$ is vector perpendicular to hyperplane. $\vec{x}_{i}$ is vector data.

$b$ is bias.

The unknown data $\vec{x}$ will compare with the hyperplane for prediction the class.

\section{Methodology}

This proposed framework is divided into 4 sections, (a) Training, (b) Object Detection, (c) Logo Detection, and (d) Evaluation and Comparison as shown in figure 13.
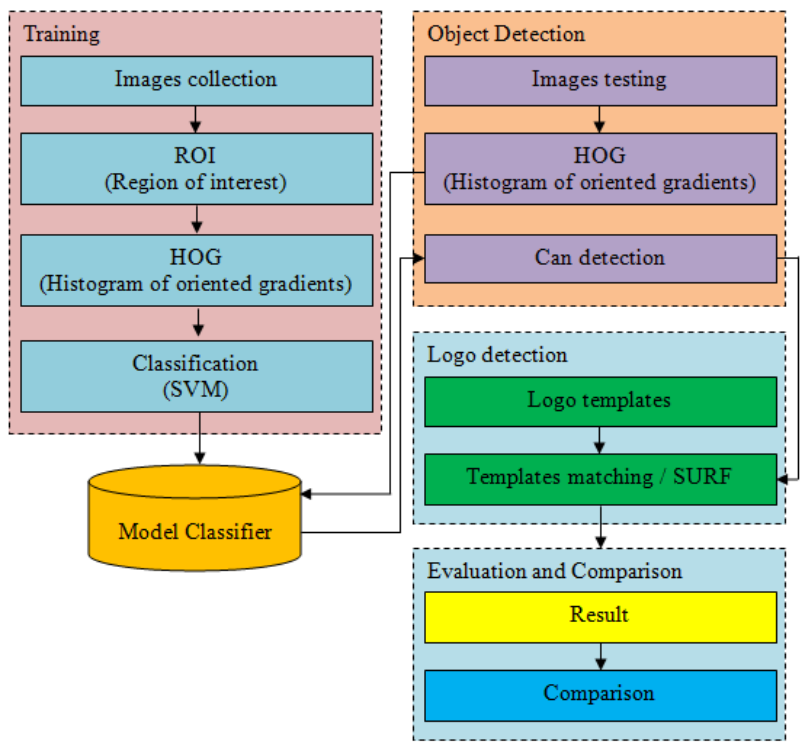

Fig. 13. Framework proposed.

(a) Training

Data classification used the training data to create a model classifier. This process is divided into 4 sections.

(i) Images collection is the process to collect the image for training set. The images are divided into 2 parts: positive and negative images. The interested object is appeared in the positive images, and unappeared in the negative image. The 100 positive image as shown in figure 14. and 20 negative images as shown in figure 15. are captured from different environments with size of images 1920x1080 pixels.
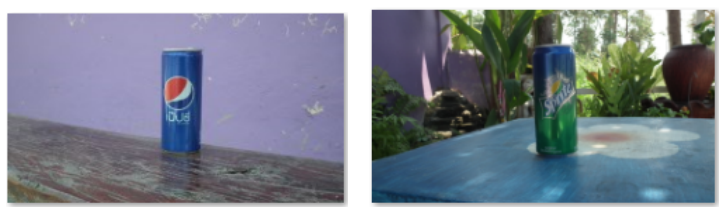

Fig. 14. Positive images for training. 

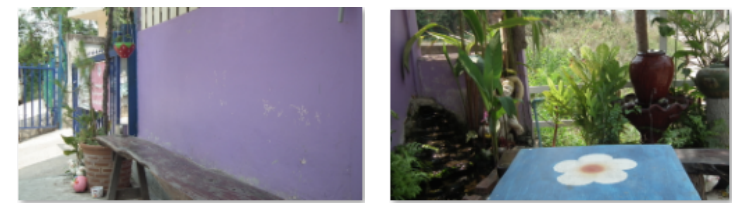

Fig. 15. Negative images for training.

(ii) Region of interest (ROI) is the selection a specifically interested region to create the training of the positive images. ROI is consisted of the point keeping the 4 values: $\mathrm{x}$-axis, $\mathrm{y}$-axis, width, and height as shown in figure 16.

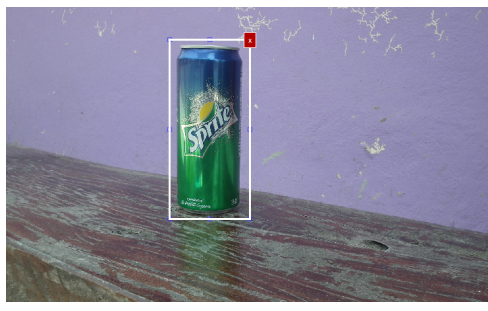

Fig. 16. Region of interest (ROI).

(iii) Histogram of oriented gradients (HOG) is used for feature extraction.

1) Gradient computation can be calculated from the $\mathrm{x}$-axis and $\mathrm{y}$-axis vector that used for convolution with kernel filter as shown in figure 17.

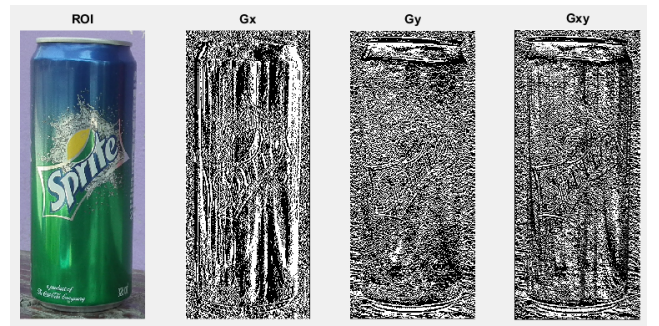

Fig. 17. Shown gradient computation.

2) Orientation binning value calculated from orientation of the gradient is stored in the bin as shown in figure 18. The orientation is defined for 0-360 degrees as shown in figure 19.
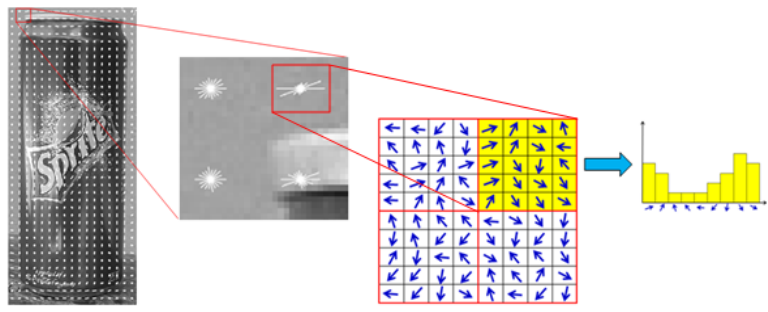

Fig. 18. Shown gradient is stored into bin.

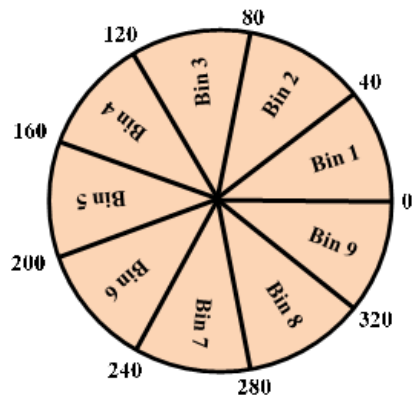

Fig. 19. The 0-360 degrees orientation.

3) Descriptor blocks are the feature description for vectors by overlapping block method as shown in figure 20.

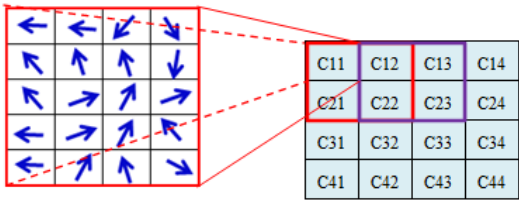

\begin{tabular}{|c|c|c|c|c|c|c|c|c|c|}
\hline \multicolumn{4}{|c|}{ Block 1} & \multicolumn{4}{|c|}{ Block 2} & $\ldots$ & Block N \\
\hline C11 & $\mathrm{C}_{12}$ & $\mathrm{C} 21$ & $\mathrm{C} 22$ & $\mathrm{C} 12$ & $\mathrm{C} 13$ & $\mathrm{C}_{22}$ & $\mathrm{C} 23$ & $\ldots$ & Cmn \\
\hline
\end{tabular}

Fig. 20. Descriptor blocks.

4) Block normalization is used the $L 1-$ norm for histograms normalization.

(iv) Support Vector Machine (SVM) algorithm is used to build classification model.

(b) Object Detection

In this process, the can detection is used in the experiment detecting by HOG. This process is divided into 3 sections.

(i) The image is separated into 2 groups: 120 images for training set and 30 images for test set. The images are consisted of 3 different sizes: small size (320x180 pixels), medium size $(640 \times 360$ pixels) and large size $(960 \times 540$ pixels) as shown in figure 21 .

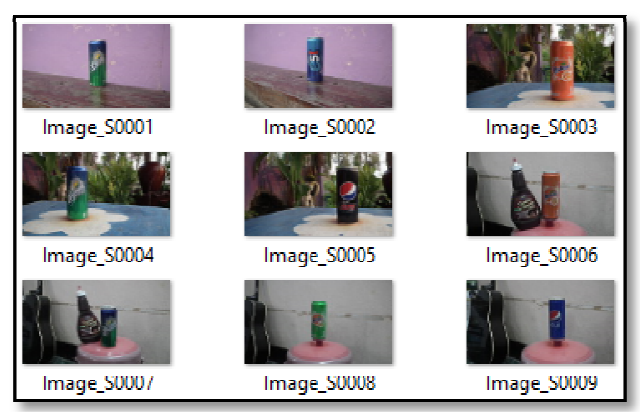

Fig. 21. Shown image testing. 
(ii) HOG is used to extract the feature.

(iii) The output of can detection is successfully using sliding window of HOG as shown in figure 22.

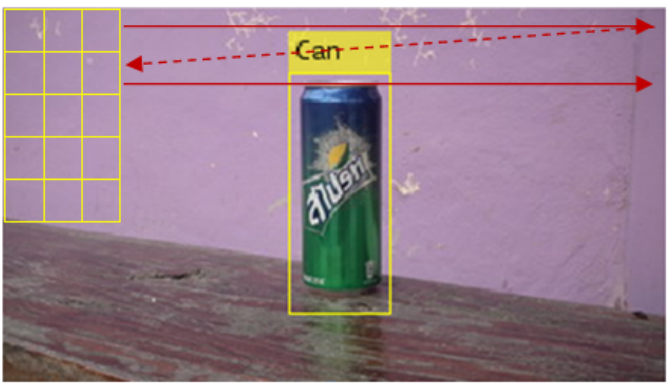

Fig. 22. Sliding window of HOG for can detection.

\section{(c) Logo Detection}

The logo detection can be found at the region of the cans this process is divided into 2 sections.

(i) The logo templates are came from cutting the logo image from cans as shown in figure 23.
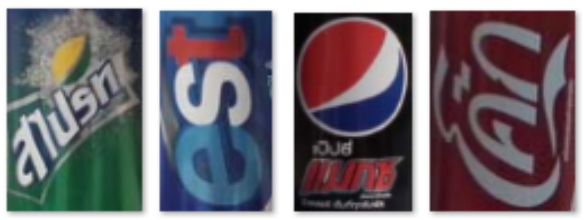

Fig. 23. Logo templates.

(ii) This research used and compared the speed of logo detection classifications that are template matching and SURF algorithm.

The output of logo detection is successfully using template matching algorithm as shown in figure 24, and SURF algorithm as shown in figure 25 .
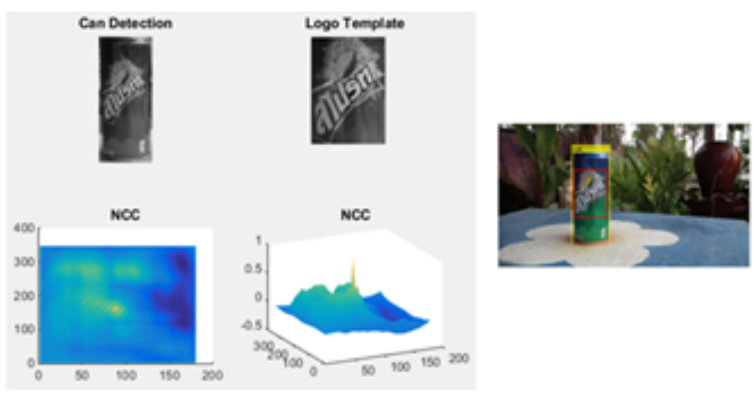

Fig. 24. Logo detection using template matching.

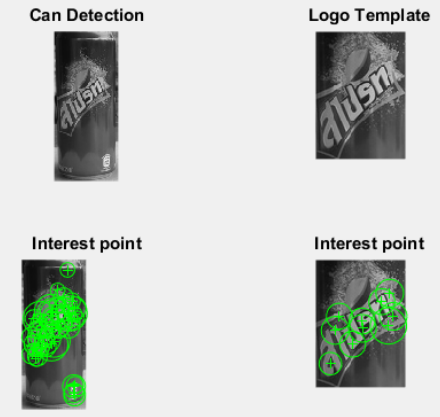

(a) Shown interested point detection.

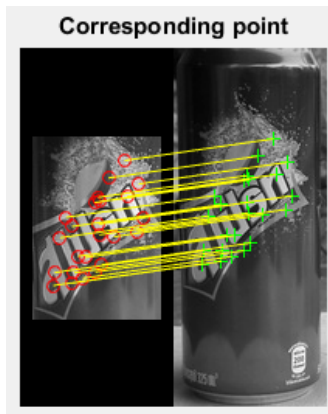

(b) Shown corresponding point

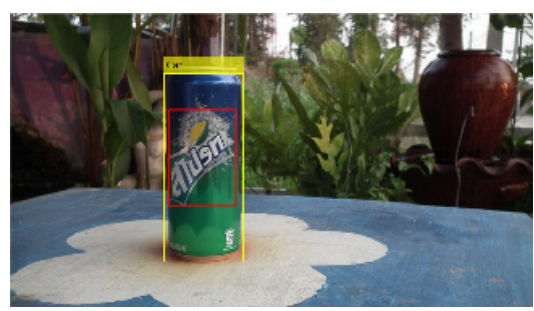

(c) Shown logo detection.

Fig. 25. Logo detection using SURF.

(d) Evaluation

The effective measurement is separated into 2 sections.

(i) The accuracy measurement is using the confusion matrix table for classification between 2 classes $^{(14)}$ for can detection as shown in table 1 .

Table 1. Confusion matrix for classification 2 class.

\begin{tabular}{|c|c|c|c|}
\hline \multicolumn{2}{|c|}{} & \multicolumn{2}{c|}{ Predicted } \\
\cline { 3 - 4 } \multicolumn{2}{|c|}{} & Negative & Positive \\
\hline$\overline{\widetilde{J}}$ & Negative & a & b \\
\cline { 2 - 4 } & Positive & c & d \\
\hline
\end{tabular}

when

$\mathrm{a}$ is the number of correct negative predictions. $\mathrm{b}$ is the number of incorrect positive predictions. $\mathrm{c}$ is the number of incorrect negative predictions. $\mathrm{d}$ is the number of correct positive predictions. 
The accuracy rate can be calculated by the equation (8).

$$
\text { Accuracy rate }=\frac{\mathrm{a}+\mathrm{d}}{\mathrm{a}+\mathrm{b}+\mathrm{c}+\mathrm{d}}
$$

(iv) The computational time is used to compare the logo detection between template matching and SURF algorithms. The experimental images is consisted of 3 sizes: small size (320x180 pixels), medium size (640x360 pixels) and large size ( $960 \times 540$ pixels).

\section{Experimental Results}

The image dataset is separated into 2 groups of images: training set, and test set. The effective measurement is separated into 2 sections.

(a) Accuracy Rate

The accuracy measurement is used to measure the accuracy of can detection by confusion matrix table as shown in table 2. The experimental result found that the accuracy is equal to $88.00 \%$.

Table 2. Confusion matrix.

\begin{tabular}{|c|c|c|c|}
\hline \multicolumn{2}{|c|}{} & \multicolumn{2}{c|}{ Predicted } \\
\cline { 3 - 4 } \multicolumn{2}{|c|}{} & Negative & Positive \\
\hline \multirow{己}{\overparen{Z}}{} & Negative & 7 & 3 \\
\cline { 2 - 4 } & Positive & 2 & 28 \\
\hline
\end{tabular}

(b) Computation time

The computational time is used for the logo detection by comparing between template matching and SURF algorithms. The experimental results as shown in figure 26 .

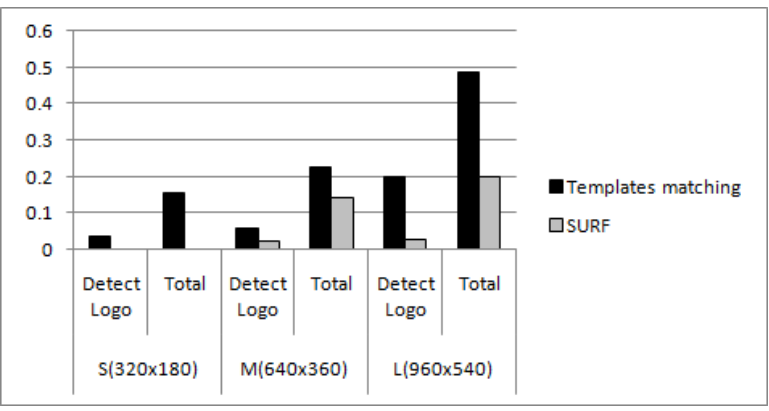

Fig. 26. Experimental result of logo detection.

\section{Conclusions}

This paper proposes the fast logo classification on the cans. The search space for logo detection is reduced by can detection using HOG. The logo detection workspace is only at the region of the cans, and computational time is came from comparison between template matching and SURF algorithm. The experimental results found that accuracy of can detection using HOG algorithm is equal to $88.00 \%$, and the computational time of logo detection found that SURF algorithm is faster than template matching.

\section{Future Enhancement}

The future research for increasing speed of logo detection is divided into 2 interested points: The modification processed of SURF algorithm, and reduction of search spec.

\section{Acknowledgment}

This research is supported by Mahasarakham University Development Fund.

\section{References}

(1) Beeran K., Suhaili et al : "Evaluation of canny and sobel operator for logo edge detection", Technology Management and Emerging Technologies (ISTMET), International Symposium on. IEEE, pp.153-156, 2014

(2) Alaei A., and Delalandre M. : "A Complete Logo Detection/Recognition System for Document Images", Document Analysis Systems (DAS), IAPR International Workshop on. IEEE, pp.324-328, 2014

(3) Sotheeswaran A., and Ramanan A. : "A classifier-free codebook-based image classification of vehicle logos", Industrial and Information Systems (ICIIS), International Conference on. IEEE, pp.1-6, 2014

(4) Kalaiyarasi C., Karthikeyan S. : "Enhancing logo matching and recognition using local features", In Information Communication and Embedded Systems (ICICES), International Conference on. IEEE, pp. 1-6, 2014

(5) Juan L., and Gwun O. : "A comparison of sift, pca-sift and surf', International Journal of Image Processing (IJIP), pp. 143-152, 2009

(6) Dimarco P. : "Logo design for record company", Computer graphics. pp.1-5, 2014

(7) Teng L. : "Package design_package design in current consumer goods", pp104, 1987

(8) Dalal N., Triggs B. : "Histograms of oriented gradients for human detection", Computer Vision and Pattern Recognition, CVPR 2005 IEEE Computer Society Conference on, pp. 886-893, 2005 
(9) Sarvaiya J., Suprava P., and Salman B. : "Image registration by template matching using normalized cross-correlation", Advances in Computing, Control, \& Telecommunication Technologies, International Conference on. IEEE, pp.819-822, 2009

(10) Bay H., Ess A., Tuytelaars T., and Gool L. "Speeded-up robust features (SURF)". Computer vision and image understanding, pp. 346-359, 2008

(11) Juan L., Gwun O. : "A comparison of sift, pca-sift and surf", International Journal of Image Processing (IJIP), pp.152-143, 2009

(12) Meyer D., Wien F. : "Support vector machines. The Interface to libsvm in package e1071", 2014

(13) Singh P., Husain M. : "Methodological Study of Opinion Mining and Sentiment Analysis Techniques", International Journal on Soft Computing, pp. 11-21. 2014

(14) Visa S., Ramsay B., Ralescu A., and Knaap E. : "Confusion Matrix-based Feature Selection". pp. $120-127,2011$ 